\title{
LncRNA CASC11 promotes the cervical cancer progression by activating Wnt/beta-catenin signaling pathway
}

\author{
Wenchan Hsu ${ }^{1,2}$, Lifen Liu' ${ }^{1}$ Xin Chen ${ }^{1}$, Ying Zhang ${ }^{1}$ and Weipei Zhu ${ }^{1 *}$
}

\begin{abstract}
Background: Studies have shown that cancer susceptibility candidate 11 (CASC11), a newly discovered long noncoding RNA (IncRNA), was aberrantly overexpressed in hepatic carcinoma, gastric cancer and colorectal cancer. However, its effects on cervical cancer has been kept unknown up to now. The present study was aimed to investigate the relationship between IncRNA CASC11 and cervical cancer and further explore the mechanism of CASC11 effect on cervical cancer progression.
\end{abstract}

Materials: Quantitative real-time polymerase chain reaction (RT-qPCR) was used to detect the expressions of CASC11 in cancerous and adjacent normal tissues of patients with cervical cancer as well as in cell lines. The proliferation, migration, invasion and apoptosis were assayed after transfecting the cell with si-CASC11 or pcDNA3.1-CASC11. TOP/ FOP-Flash luciferase reporter assay and western blot were used to analysis the activation of $\mathrm{Wnt} / \beta$-catenin signaling pathway. Si-CASC11-transfected HeLa cells were subcutaneously inoculated into male athymic (nude) mice to investigate the effect of CASC11 on the tumor formation.

Results: We discovered that CASC11, the expression of which was positively associated with the tumor size and the FIGO staging and negatively related to the patients' survival rate, was up-regulated in the cervical cancer tissues and cell lines. Silencing CASC11 inhibited the proliferation, migration as well as invasion and promoted the cell apoptosis. Conversely, overexpression of CASC11 facilitated the cancer cell's proliferation, migration and invasion ability and suppressed the apoptosis. Further study showed that CASC11 promoted the migration and invasion of cervical cancer cells by activating Wnt/ $\beta$-catenin signaling pathway and silencing CASC11 inhibited the tumor growth in vivo.

Conclusion: Our study demonstrated that CASC11 promoted the cervical cancer progression by activating Wnt/ $\beta$ catenin signaling pathway for the first time, which provides a new target or a potential diagnostic biomarker of the treatment for cervical cancer.

Keywords: LncRNA, CASC11, Cervical cancer, Wnt/ß-catenin

\section{Background}

Cervical cancer is one of the most common malignant tumors in women worldwide with high morbidity and mortality. Numbers of factors, such as genetic influences and viral infection, are involved in this complex disease. It is well known that cervical cancer is developed from cervical intraepithelial neoplasia (CIN), in which

\footnotetext{
*Correspondence: zwp333xx@aliyun.com

1 Department of Gynecology and Obstetrics, The Second Affiliated

Hospital of Soochow University, Suzhou 215004, China

Full list of author information is available at the end of the article
}

the infection of human papillomavirus (HPV) plays an initial role in the progression of the cervical lesions [1]. However, studies have shown HPV infection alone is not a sufficient condition for cervical cancer [2]. Specific chromosomal changes mainly including abnormal amplifications of chromosome $3 \mathrm{q}$ and protooncogene MYC (8q24) also occurred in malignant cervical lesions [3]. Meanwhile, recent researches showed that there may exist a close relationship between HPV protein and long non-coding RNA (lncRNA) in cervical cancer [4]. 
Unfortunately, the pathogenesis of cervical cancer has not been fully understood up to now.

LncRNA is a class of single-stranded RNAs with a length of more than 200 nucleotides (nt) and without the capacity of coding proteins. It works in cell growth, survival, migration, invasion, differentiation, and other cellular processes. The abnormal expression or dysfunction of lncRNA is closely related to the occurrence of cancers [5]. With the development of molecular biology technology, many lncRNAs were found to be served as potential and minimally invasive diagnostic/prognostic biomarkers, and also as therapeutic targets for cancers. For example, IncPCA3 has been routinely used for the diagnosis of prostate cancer in clinic [6]. More than a dozen of lncRNAs including HOX transcript antisense RNA (HOTAIR), metastasis associated lung adenocarcinoma transcript 1 (MALAT1), colon cancer-associated transcript 2 (CCAT2) and so on, are involved in cervical cancer by interacting with proteins, miRNAs and other molecules, providing accurate targets for the treatment of cervical cancer patients [7].

In recent years, researchers have found a variety of cancer-related lncRNAs localized on chromosome 8q24. CCAT2, which was located on 8q24.21 and originally identified as an oncogenic lncRNA in colorectal cancer, was significantly up-regulated in many kinds of malignant tumors containing lung cancer, colorectal cancer, breast cancer, gastric cancer, cervical cancer and so on, indicating that CCAT2 might be a therapeutic target [8]. Cancer susceptibility candidate 11 (CASC11), which was also located on 8q24.21 region, not only promoted the growth and metastasis of colorectal cancer cells by interacting with heterogeneous ribonucleoprotein $\mathrm{K}$ (hnRNP-K) and activating Wnt/ $\beta$-catenin pathway [9], but also facilitated the cell proliferation, migration and invasion in hepatocellular carcinoma and in gastric cancer via activating PI3K/AKT signaling pathway and regulating cell cycle pathway, respectively $[10,11]$. However, no one has yet reported the effects of lncRNA CASC11 in cervical cancer.

Wnt/ $\beta$-catenin pathway is a classical signaling pathway involved in the development and progression of cancers. It initiates the transcription of the downstream target genes through activating $\beta$-catenin. Studies have shown that the aberrant activation of the $\mathrm{Wnt} / \beta$-catenin pathway is closely related to HPV-infected cervical cancer [12]. In the present study, we explored the relationship between lncRNA CASC11 and cervical cancer finding that high expression of IncRNA CASC11 promoted the growth and metastasis of cervical cancer through Wnt/ $\beta$-catenin pathway, which provided a new target for the treatment of cervical cancer.

\section{Materials and methods}

\section{Tissue samples collection}

Cancerous and adjacent normal tissues of 50 patients with cervical cancer who had not received prior treatment were obtained from the Second Affiliated Hospital of Soochow University from 2015 to 2018. All patients signed the informed consent. The samples were immediately frozen in liquid nitrogen and stored at $-80{ }^{\circ} \mathrm{C}$. The pathological classification and clinical stages were performed to the International Federation of Gynecology and Obstetrics (FIGO) criteria. All patients signed the informed content. All the protocols in this study were approved by the Ethics Committee of the Second Affiliated Hospital of Soochow hospital.

\section{Cell culture and processing}

Human foreskin keratinocytes (HEKn, Cat. no. C-001-5C) were obtained from the Cascade BiologicsTm (Portland, OR, USA) and cultured in EpiLife medium (Gibco/LifeTechnologies, Waltham, MA; Cat. No. M-EPI-500-CA). The human cervical cancer cell lines (HeLa, CaSki, SiHa, C-33A and MS751) were purchased from Cell Bank of the Chinese Academy of Science (Shanghai, China) maintaining in RPMI1640 medium. All the mediums were supplemented with $10 \%$ fetal bovine serum (FBS; Hyclone; Invitrogen, Camarillo, CA, USA) and $100 \mathrm{U} / \mathrm{ml}$ penicillin/100 $\mu \mathrm{g} / \mathrm{ml}$ streptomycin (Invitrogen, Carlsbad, CA, USA) at $37^{\circ} \mathrm{C}$ with $5 \% \mathrm{CO}_{2}$. For the cell processing, $10 \mathrm{mM} \mathrm{LiCl}$ or $100 \mathrm{ng} / \mathrm{ml}$ Dkk-1, which are served as the Wnt signaling pathway activator and inhibitor respectively, were added in to the medium $36 \mathrm{~h}$ after cell transfection.

\section{Subcutaneous xenografts in mice}

As previously described [13], $4 \times 10^{6} \mathrm{HeLa}$ cells, which were transfected with si-CASC11 and si-NC for $24 \mathrm{~h}$, were subcutaneously inoculated into male athymic nude mice ( $n=12,6-8$ weeks old). The tumors' size was measured every 5 days with calipers and the volume of the tumors were calculated as length $\times\left(\right.$ width $\left.^{2}\right) / 2.35$ days after affections, the tumors were removed surgically and measured the weight.

\section{Quantitative real-time polymerase chain reaction (RT-qPCR)}

According to the manufacturer's instructions, total RNA was extracted from cells with RNAiso Plus (Code No. 9108, TaKaRa, Dalian, China). According to the protocols of manufacturer, RNA quality was assessed using a NanoDrop 2000 Spectrophotometer (Thermo Scientific). Then $1 \mu \mathrm{g}$ total RNA was converted into the first strand cDNA using PrimeScript RT reagent kit (Takara, Tokyo, 
Japan). Finally, the PCR amplification was performed using a SYBR ${ }^{\circledR}$ Premix Ex Taq ${ }^{\mathrm{TM}}$ II kit (Code No. RR820A, TaKaRa). The reaction system $(20 \mu \mathrm{l})$ included $10 \mu \mathrm{l}$ TB Green Premix Ex Taq II (Tli RNaseH Plus, $2 \times$ ), $0.8 \mu \mathrm{l}$ PCR Forward Primer $(10 \mu \mathrm{M}), 0.8 \mu \mathrm{l}$ PCR Reverse Primer $(10 \mu \mathrm{M}), 0.4 \mu \mathrm{l}$ ROX Reference Dye $(50 \times), 2 \mu \mathrm{l}$ DNA template and $6 \mu \mathrm{l}$ sterile water. The PCR reaction conditions as follows: $95^{\circ} \mathrm{C}$ for $30 \mathrm{~s}$ followed by 40 cycles at $95^{\circ} \mathrm{C}$ for $5 \mathrm{~s}$ and $60^{\circ} \mathrm{C}$ for $30 \mathrm{~s}$. After the cycling protocol, the final step was applied to all reactions by continuously monitoring fluorescence through the dissociation temperature of the PCR product at a temperature transition rate of $5{ }^{\circ} \mathrm{C}$ to generate a melting curve, and then Cooling $50{ }^{\circ} \mathrm{C}$ for $30 \mathrm{~s}$. Quantification was conducted according to the $2^{-\Delta \Delta c t}$ method. The relative expression of CASC11 was analyzed in an Applied Biosystems 7500 Fast real-time PCR system and was normalized with glyceraldehyde3-phosphate dehydrogenase gene (GAPDH). The primers were purchased from Sangon Biotech (Shanghai, China), and the sequences were shown as follows: CASC11 sense 5'-GCTGCAGA AGGTCCGAAGAA-3', CASC11 antisense 5'-TTCACCACGTCCAGTTGCTT-3'; GAPDH sense $5^{\prime}$-GGAGCGAGATCCCTCCAAAAT-3, GAPDH antisense: 5'-GGCTGTTGTCATACTTCTCATGG-3'.

\section{Cell transfection}

For optimal siRNA transfection efficiency, siRNA sequences were designed to target the human CASC11 gene and the CASC11 siRNAs and control siRNAs were obtained from GenePharma (Shanghai GenePharma Co., Ltd., Shanghai, China). The sequences of siRNAs were 5'-GCCCACATCAAGCCTTCAT-3' (CASC11 siRNAs) and $5^{\prime}$-UUCUCCGAACGUGUCACGU-3' (control siRNAs). Cells $\left(2 \times 10^{5}\right.$ cells/well $)$ were added in 6-well plates and transfected with CASC11 siRNAs (100 nM) or control siRNAs $(100 \mathrm{nM})$ for $48 \mathrm{~h}$ using Lipofectamine ${ }^{\mathrm{TM}}$ 2000 (Invitrogen; Thermo Fisher scientific, Inc.) according to the instructions.

A CASC11 overexpression plasmid, pcDNA3.1CASC11, was commercially constructed by GenePharma (Shanghai, China), and empty pcDNA 3.1 vector $(\mathrm{NC})$ was used as the control. To establish cell lines with transient overexpression of CASC11, and CaSki cells $\left(5 \times 10^{5}\right.$ / $\mathrm{ml}$ ) were seeded into 6-well plates and transfected with $10 \mu \mathrm{g}$ pcDNA3.1-CASC11 plasmid or control pcDNA3.1 vector in medium using lipofectamine ${ }^{\mathrm{TM}} 2000$ (Invitrogen). And the effect of CASC11 silencing and overexpression is shown in Additional file 1: Fig. S1.

\section{Cell Counting Kit-8 (CCK-8) assay}

Cell Counting Kit-8 (CCK-8, Solarbio, China) assay was used to analyze the cell proliferation. Briefly, $100 \mu \mathrm{l}$ cell suspension were seeded into 96-well plates. $0 \mathrm{~h}, 12 \mathrm{~h}$, $24 \mathrm{~h}, 48 \mathrm{~h}$ or $96 \mathrm{~h}$ after cell transfection, $10 \mu \mathrm{l}$ CCK-8 reagent was added into the medium incubating in dark at $37{ }^{\circ} \mathrm{C}$ for $2 \mathrm{~h}$. Finally, the absorbance at $450 \mathrm{~nm}$ was determined with a full wavelength multifunctional enzyme labeling apparatus (TECAN).

\section{Flow cytometric analysis for apoptosis}

$48 \mathrm{~h}$ after cell transfection, the apoptosis of HeLa cells and CaSki cells was measured with an Annexin V-FITC/ PI apoptosis detection kit (BD Biosciences) following the manufacturer's instructions. The apoptosis rate was analyzed using a FACSCalibur ${ }^{\mathrm{TM}}$ flow cytometer (BD Biosciences) as previously mentioned [11].

\section{Transwell assay for cell invasion and migration}

Transwell Chambers (Corning) uncoated or coated with Matrigel were used for assessing the invasion and migration of cervical cancer cells. The detailed methods were as mentioned as Lan described [14]. The migratory or invasive cells was imaged and counted utilizing an optical microscope (Carl Zeiss, Jena, Germany).

\section{TOP/FOP-Flash luciferase reporter analysis}

TOP/FOP-Flash luciferase reporter assay was used to analyze the activity of $\mathrm{Wnt} / \beta$-catenin signaling pathway. The vector pRL-SV40 was served as the internal reference. Cells in the control groups were co-transfected with pRL-SV40 and TOP/FOP flash (Promega). Cells in NC groups were co-transfected with pRL-SV40, TOP/FOP flash and si-CASC11 NC/pcDNA 3.1. Cells in si-CASC11 group or pcDNA3.1-CASC11 group were co-transfected with pRL-SV40, TOP/FOP flash and si-CASC11 NC/ pcDNA 3.1-CASC11. Dual-Luciferase Reporter Assay System (E1910; Promega) was applied to measure the luciferase activity. The ratio of TOP/FOP indicated the activity of Wnt/ $\beta$-catenin signaling pathway.

\section{Western blot}

Total proteins were extracted from cells with RIPA lysis buffer (P0013B, Beyotime) and nuclear extracts were collected with a Nuclear and Cytoplasmic Protein Extraction Kit (P0027, Beyotime). An enhanced BCA protein assay kit (P0010, Beyotime) was used to measure the protein concentration. $50 \mu \mathrm{g}$ protein per well was separated by $10 \%$ sodium dodecyl sulfate polyacrylamide gel electrophoresis (SDS-PAGE) followed by transferred onto polyvinylidene difluoride membranes (Millipore). After blocked with 5\% non-fat milk, the bands were incubated with primary antibodies followed by horseradish peroxidase (HRP)-conjugated secondary antibodies (\#7074, 1:2000) from Cell Signaling Technology. The primary antibodies were as follows: $\beta$-catenin rabbit 
monoantibody (\#8480, 1:1000), GAPDH rabbit monoantibody (\#8884, 1:1000), Lamin B rabbit monoantibody (\#12255, 1:1000). The target proteins were visualized with a BeyoECL Plus kit (P0018, Beyotime). Densitometric analysis was carried out with Image J software.

\section{Statistical analysis}

All the experiments were replicated independently at least three times in triplicate. The data were represented as means \pm standard deviation (SD) and analyzed with one-way ANOVA using SPSS version 22.0 software (IBM, Chicago, IL, USA). The relationship between the overall survival (OS) of patients with cervical cancer and CASC11 was evaluated with the Kaplan-Meier test. A value of $p<0.05$ was considered statistically significant.

\section{Results}

\section{Up-regulation of CASCA11 expression in cervical cancer} tissues

The relative expression of CASC11 detected by RTqPCR was much higher in the tumor samples than that in the adjacent normal tissues $(\mathrm{n}=50$ tumor; $\mathrm{n}=50$ adjacent normal; Fig. 1a). The correlation between CASC11 and clinicopathological characteristics was analyzed in Table 1 . The age, menopause, lymph node metastasis and lymphatic vascular space invasion of patients have little effect on CASC11 expression, but which was positively correlated with the FIGO stage and the tumor size (Fig. 1b, c). 50 cervical cancer patients were divided into high and low CASC11 expression groups $(\mathrm{n}=25$, respectively) according to the median CASC11 expression level.
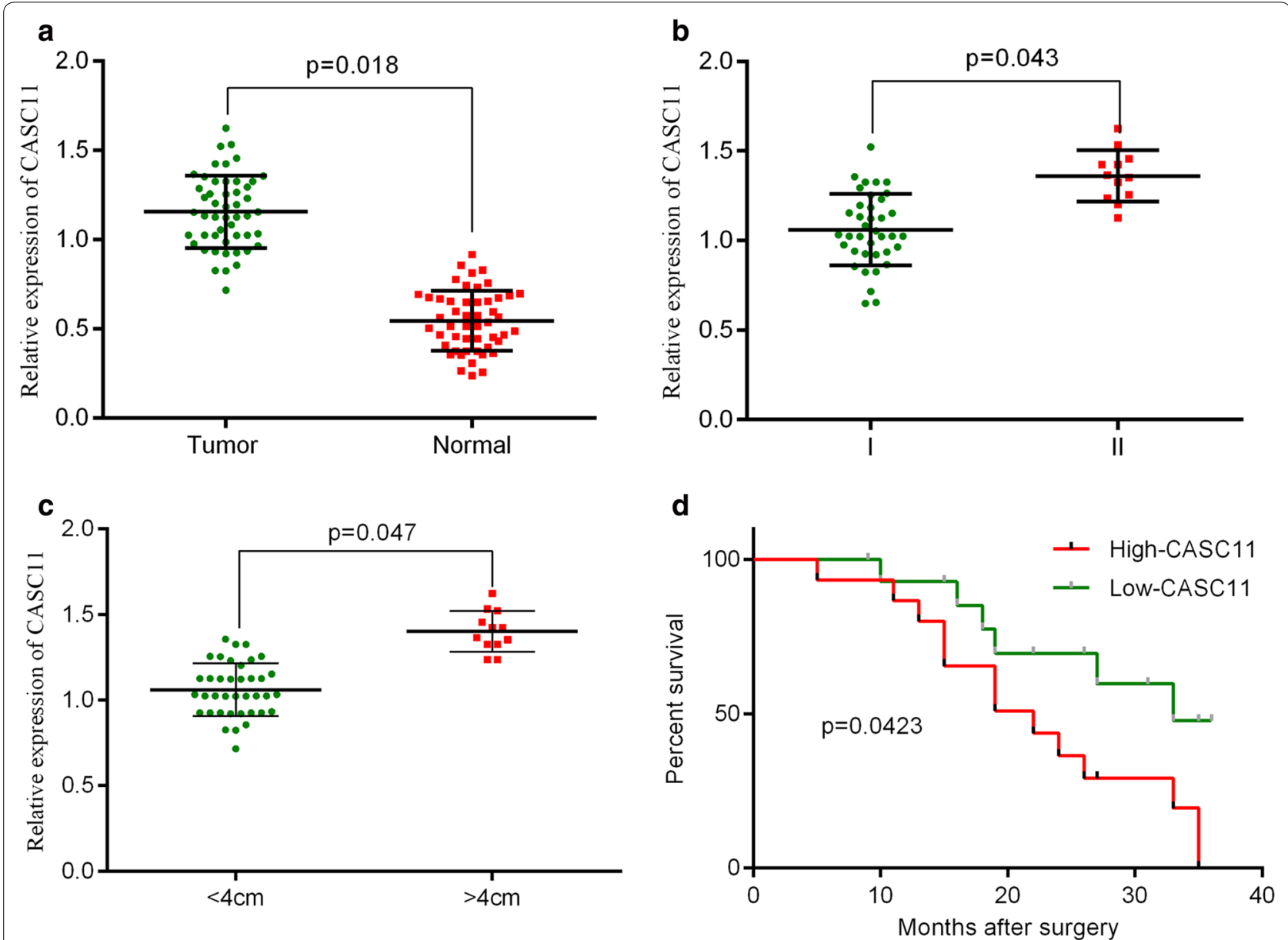

Fig. 1 Relative expression levels of InCRNA CASC11 in cervical cancer tissues. a CASC11 expression was assessed by RT-qPCR assay in cervical cancer samples $(n=50)$ and adjacent normal samples $(n=50)$, and CASC11 was up-regulated in cervical cancer. $\mathbf{b}$ The expression level of CASC11 was evaluated by RT-qPCR assay in FIGO stage I $(n=38)$ and FIGO stage II $(n=12)$, and CASC11 was positively related to the FIGO stage. $\mathbf{c}$ The expression level of CASC11 was analyzed by RT-qPCR assay in tumor tissues (tumor size $<4 \mathrm{~cm}, \mathrm{n}=38$ ) and tumor tissues (tumor size $\geq 4 \mathrm{~cm}, \mathrm{n}=12$ ), and CASC11 was positively related to the tumor size. $\mathbf{d}$ Kaplan-Meier test showed that high level of CASC11 was significantly associated with the low survival rate for cervical cancer patients. CASC11 expression was detected by RT-qPCR analysis and normalized to GAPDH expression ( $n=25$, respectively). Error bars represent the mean $\pm S D$ of 3 independent experiments. ${ }^{*} p<0.5$; ${ }^{* *} p<0.01$ 
Table 1 Correlation between CASC11 and clinicopathological characteristics $(n=50)$

\begin{tabular}{|c|c|c|c|}
\hline Clinicopathologic feature & N (\%) & CASC11 (mean \pm SEM) & $p$-value \\
\hline Age & & & 0.836 \\
\hline$\leq 60$ & $19(38 \%)$ & $1.23 \pm 0.17$ & \\
\hline$>60$ & $31(62 \%)$ & $1.17 \pm 0.20$ & \\
\hline Tumor size (cm) & & & $0.047^{*}$ \\
\hline$<4$ & $38(76 \%)$ & $1.05 \pm 0.12$ & \\
\hline$\geq 4$ & $12(24 \%)$ & $1.42 \pm 0.15$ & \\
\hline \multicolumn{4}{|l|}{ Menopause } \\
\hline Yes & $26(52 \%)$ & $1.29 \pm 0.17$ & 0.925 \\
\hline No & $24(48 \%)$ & $1.30 \pm 0.14$ & \\
\hline \multicolumn{4}{|l|}{ Lymph node metastasis } \\
\hline Negative & $23(46 \%)$ & $1.25 \pm 0.20$ & 0.918 \\
\hline Positive & $27(54 \%)$ & $1.27 \pm 0.18$ & \\
\hline \multicolumn{4}{|c|}{ Lymphativ vascular space invasion } \\
\hline Negative & $29(58 \%)$ & $1.23 \pm 0.12$ & 0.816 \\
\hline Positive & $21(42 \%)$ & $1.36 \pm 0.10$ & \\
\hline FIGO stage & & & $0.043^{*}$ \\
\hline । & $38(78 \%)$ & $1.03 \pm 0.15$ & \\
\hline ॥ & $12(24 \%)$ & $1.39 \pm 0.16$ & \\
\hline
\end{tabular}

${ }^{*} p<0.5$

Kaplan-Meier test showed that high level of CASC11 was significantly associated with the low survival rate for cervical cancer patients (Fig. 1d). We further measured the RNA levels of CASC11 in cervical cancer cells finding that the relative expression of CASC11 in HeLa, CaSki, SiHa, C-33A and MS751 were remarkably higher than that in the normal ectocervical cells HEKn. Furthermore, among the cervical cancer cell lines, HeLa cells showed the highest expression of CASC11 and CaSki cells displayed the lowest CASC11 level (Fig. 2). So, we chose

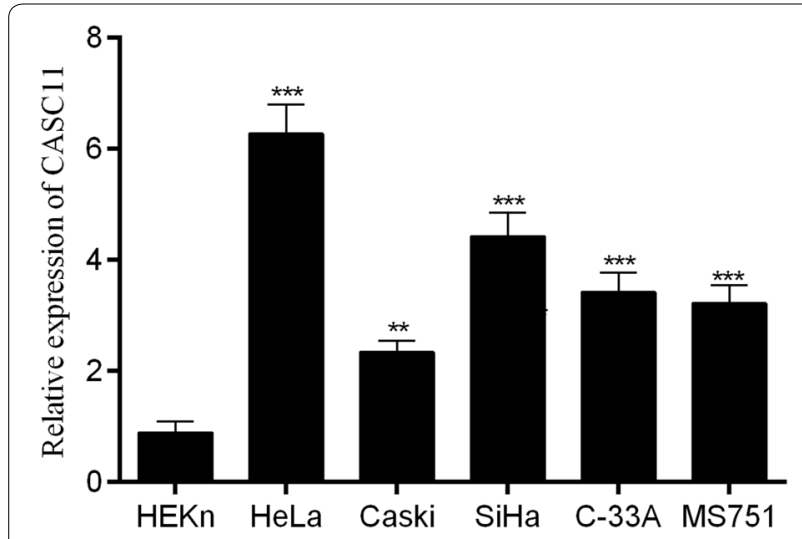

Fig. 2 CASC11 was up-regulated in cervical cancer cell lines. CASC11 expression was detected by RT-qPCR analysis in HEKn and cervical cancer cell lines (HeLa, Caski, SiHa, C-33A and MS751). The level of CASC11 was normalized to GAPDH. Error bars represent the mean \pm SD of 3 independent experiments. ${ }^{* *} p<0.01$; ${ }^{* *} p<0.001$
HeLa cells for si-CASC11 transfecting and chose CaSki cells for pcDNA3.1-CASC11 transfection.

\section{CASC11 promoted the proliferation and inhibited the apoptosis of cervical cancer cells}

Silencing CASC11 with siRNA in HeLa cells significantly decreased the cell proliferation and increased the apoptosis of cells compared to that in the control cells and the si-CASC11 NC-transfected cells. Conversely, overexpression of CASC11 by transfecting the CaSki cells with pcDNA3.1-CASC11 significantly increased the cell proliferation while decreased the apoptosis of cells versus that in control and CASC11 NC groups (Fig. 3a, b).

\section{CASC11 promoted the migration and invasion of cervical cancer cells.}

Transwell experiments showed that knockdown of CASC11 in HeLa cells showed significantly lower abilities of migration and invasion than that in control and si-CASC11 NC-transfected cells while overexpression of CASC11 in CaSki cells displayed much higher abilities of migration and invasion than that in control and CASC11 NC groups (Fig. 4a, b).

\section{CASC11 involved in the activating of Wnt/ $\beta$-catenin signaling pathway}

TOP/FOP-Flash luciferase reporter assay showed that down-regulated the expression of CASC11 in HeLa 


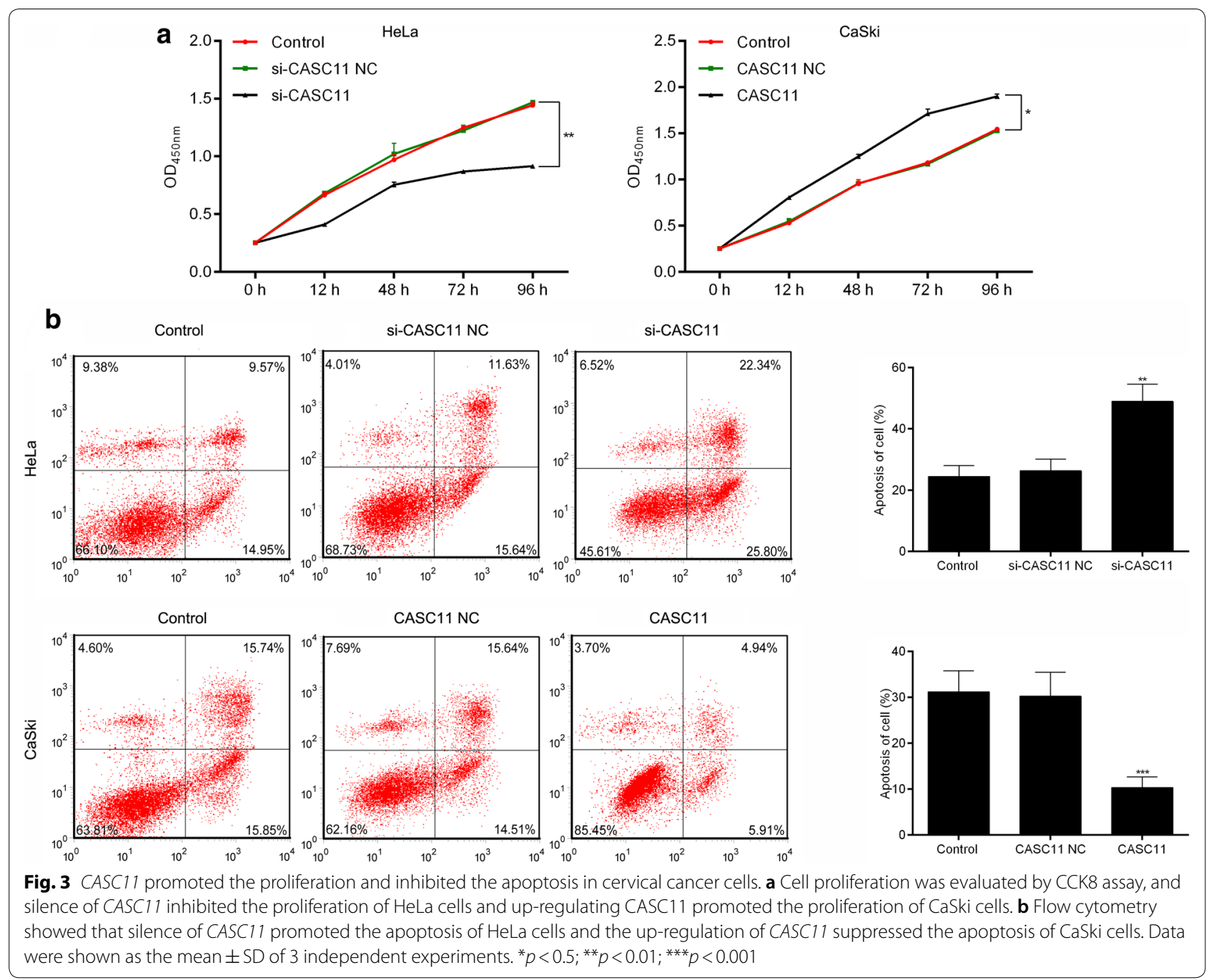

cells inhibited the activity of $\mathrm{Wnt} / \beta$-catenin signaling pathway while overexpression of CASC11 in CaSki cells significantly up-regulated the signaling activity (Fig. 5a). Western blot analysis indicated that silencing CASC11 significantly decreased the total and nuclear expressions of $\beta$-catenin, while overexpression of CASC11 observably increased the total and nuclear expressions of $\beta$-catenin (Fig. $5 \mathrm{~b}$ ). These results suggested that CASC11 was involved in the activation of $\mathrm{Wnt} / \beta$-catenin signaling pathway.

\section{CASC11 promoted the migration and invasion of cervical cancer cells through activating Wnt/ $\beta$-catenin signaling} As shown as Fig. 6a, b, the si-CASC11-transfected cells treated with the Wnt signaling activator $\mathrm{LiCl}$ or si-CASC11 NC showed a much higher ability of migration and invasion than that without $\mathrm{LiCl}$ treatment. The pcDNA3.1-CASC11 transfected cells treated with the Wnt signaling inhibitor Dkk-1 or CASC11 NC displayed a lower ability of migration and invasion than that without Dkk-1 treatment. Western blot displayed that compared to the cell only transfected with si-CASC11 or pcDNA3.1-CASC11, LiCl significantly increased the expressions of total and nuclear $\beta$-catenin and Dkk-1 significantly decreased the total and nuclear $\beta$-catenin expressions (Fig. 7a, b). Therefore, these results indicated that $C A S C 11$ requires an active $\mathrm{Wnt} / \beta$-catenin pathway to contribute to the phenotype.

\section{Knock-down CASC11 inhibited the proliferation of tumor in vivo}

As shown as Fig. 8a, b, the tumor volume and weight of mice in the HeLa/si-CASC11 group were bigger and heavier than that in the HeLa/si-CASC11NC group. As expected, western blot analysis showed that the expression of $\beta$-catenin in the HeLa/si-CASC11 group was much higher than that in the HeLa/si-CASC11 

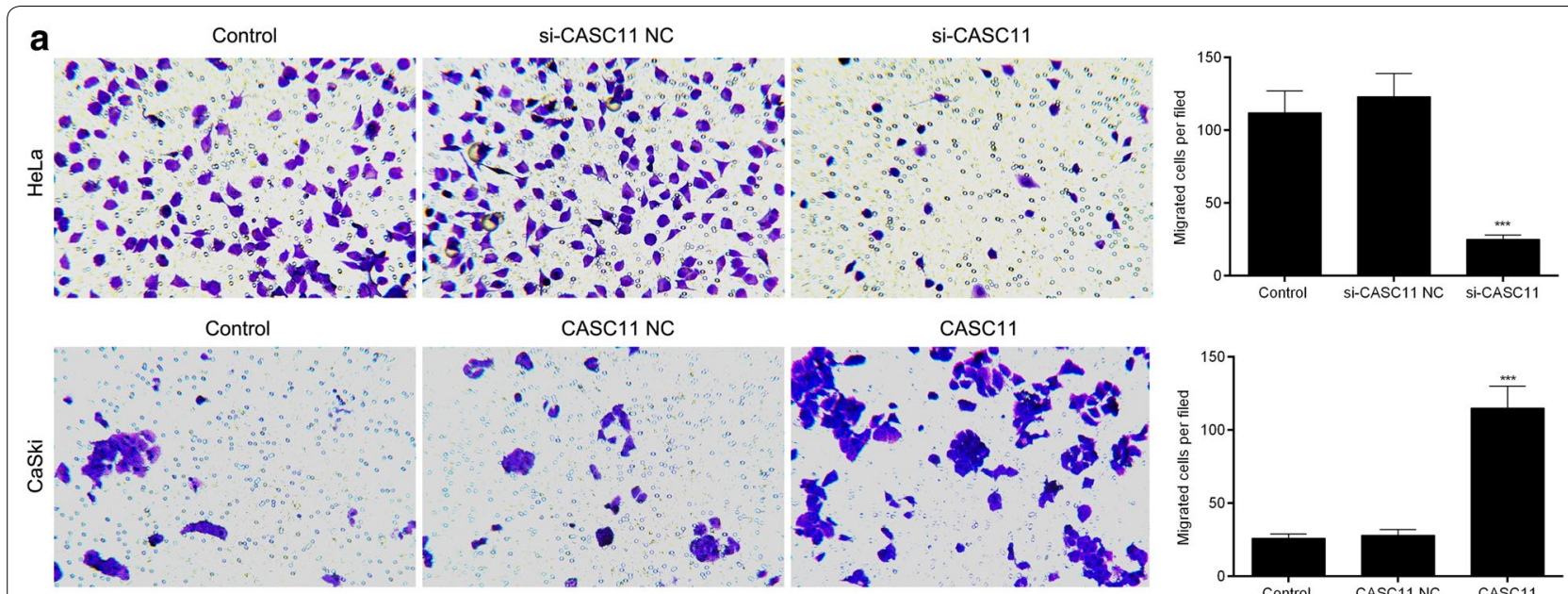

b Control

si-CASC11 NC
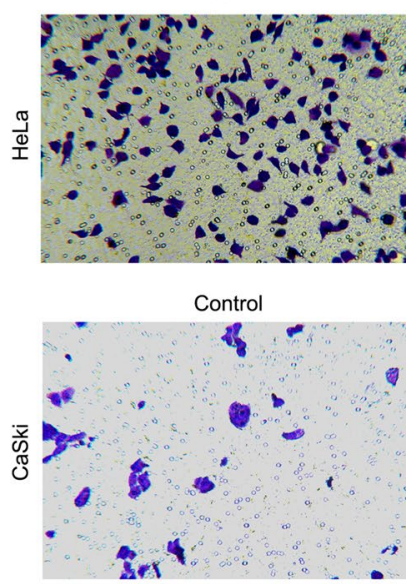

CASC11 NC
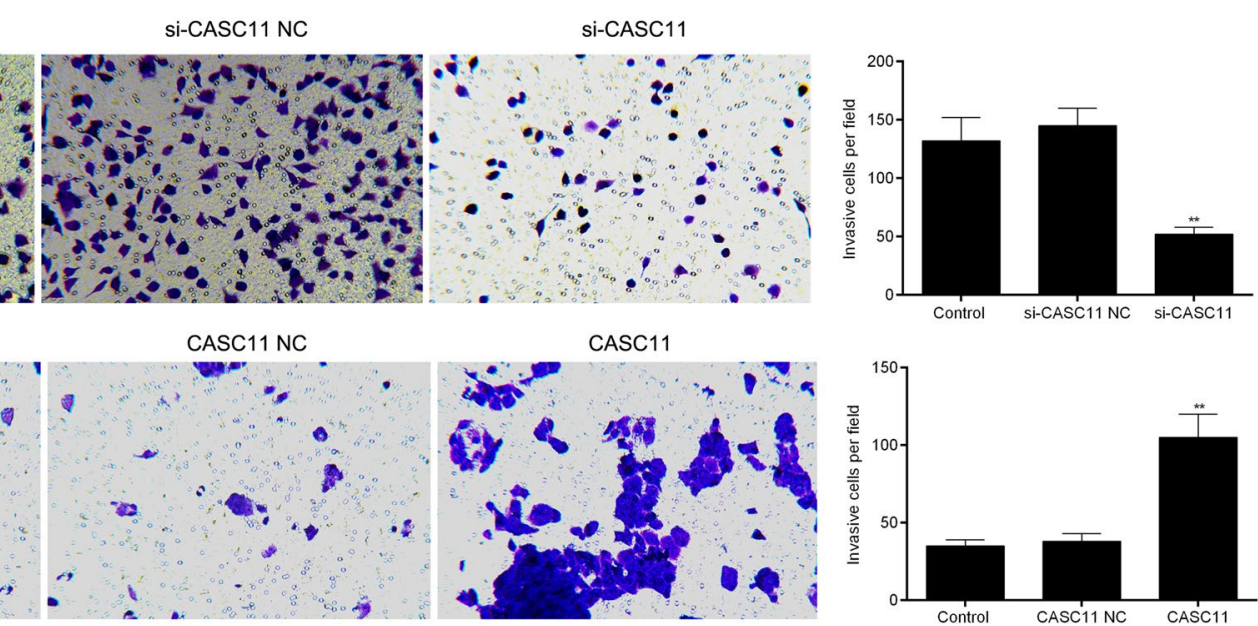

Fig. 4 CASC11 promoted the migration and invasion in cervical cancer cells. a Transwell assay showed that silencing CASC11 inhibited the migration of HeLa cells and up-regulating CASC11 promoted the migration of CaSki cells. $\mathbf{b}$ Cell invasion was measured by Transwell assay, and silencing CASC11 inhibited the invasion of HeLa cells and up-regulating CASC11 promoted the invasion of CaSki cells. Data were shown as the mean \pm SD of 3 independent experiments. ${ }^{* *} p<0.01 ;{ }^{* * *} p<0.001$

group (Fig. 8c). These data hints that CASC11 promoted the proliferation of tumor in vivo.

\section{Discussion}

Chromosome 8q24, which is about $600 \mathrm{~kb}$ in length, was once thought to be a gene desert. Genome-wide association study (GWAS) has shown that genetic mutations in 8q24 region are associated with the risk of prostate cancer, colorectal cancer and other malignant tumors [15]. Another study indicated that MYC (8q24) played a vital part in the progression of CIN to cervical cancer [3]. Recently, Shen et al. [16] found that in HeLa cell line, the integrated HPV, MYC and 8q24.22 was close to each other, which might form a trimer in spatial location promoting the development of cervical cancer. CASC11 is a newly discovered lncRNA $\sim 2.1 \mathrm{~kb}$ upstream of c-Myc in chromosome 8q24 gene desert. The upregulation of CASC11 was reported to participated in the proliferation, migration and invasion of tumor cells in hepatic carcinoma, gastric and colorectal cancer. Zhang et al. [11] found that c-Myc could directly bind to the CASC11 promoter regions increasing the promoter histone acetylation to enhance CASC11 expression in colorectal cancer. Therefore, we hypothesized that $C A S C 11$ has a similar function for cervical cancer and discovered that CASC11 promoted the proliferation, metastasis and invasion of cervical cancer cells through $\mathrm{Wnt} / \beta$-catenin signaling pathway. 

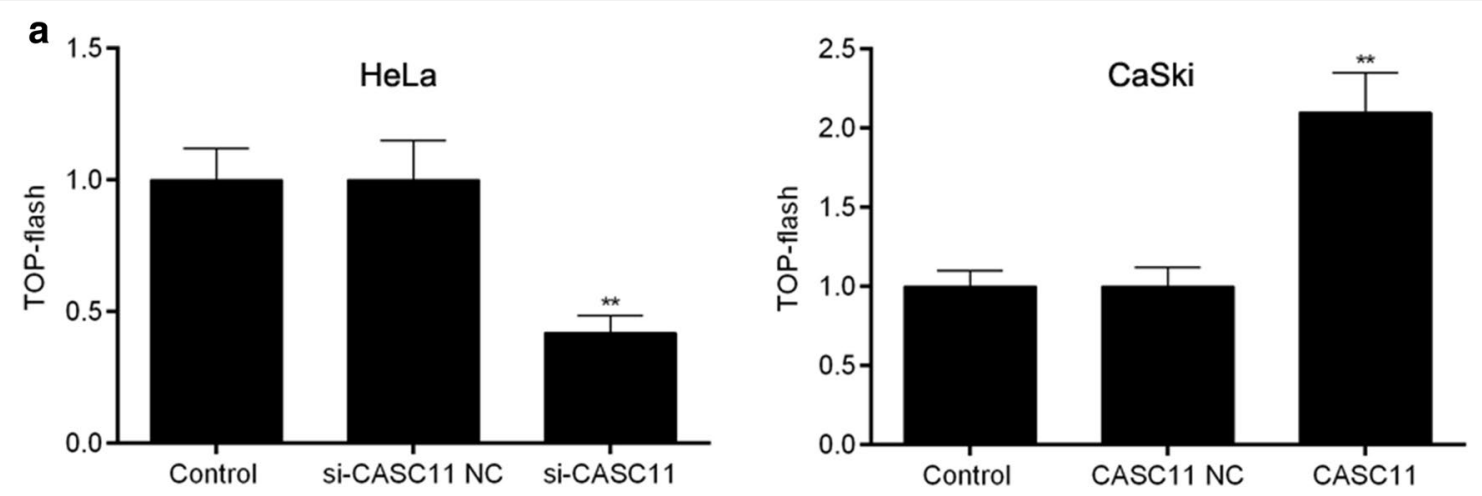

b

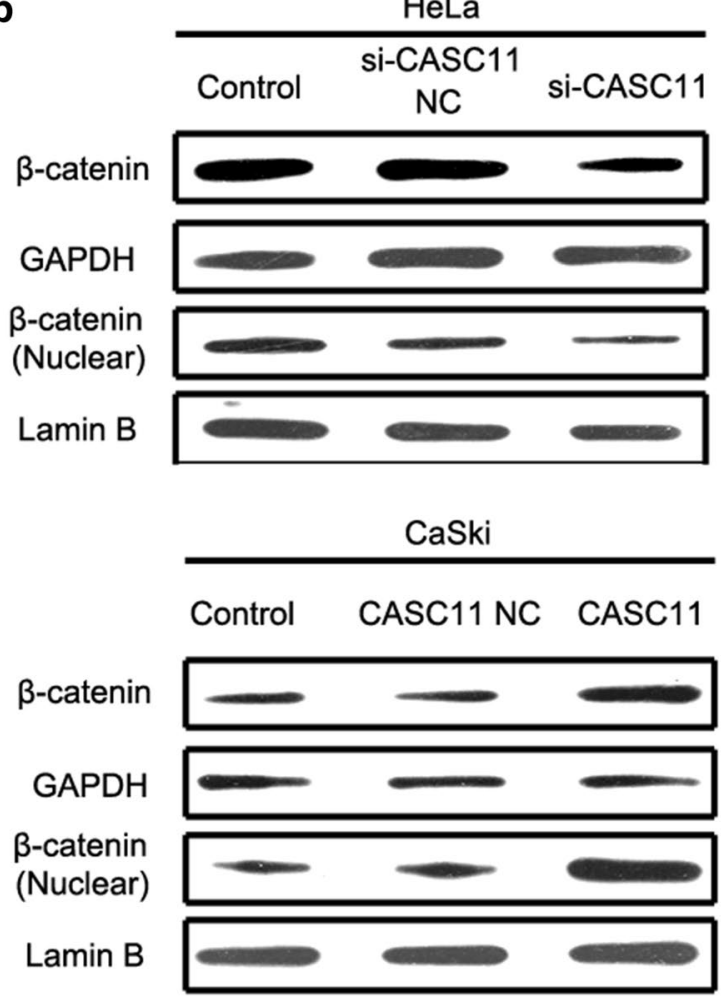

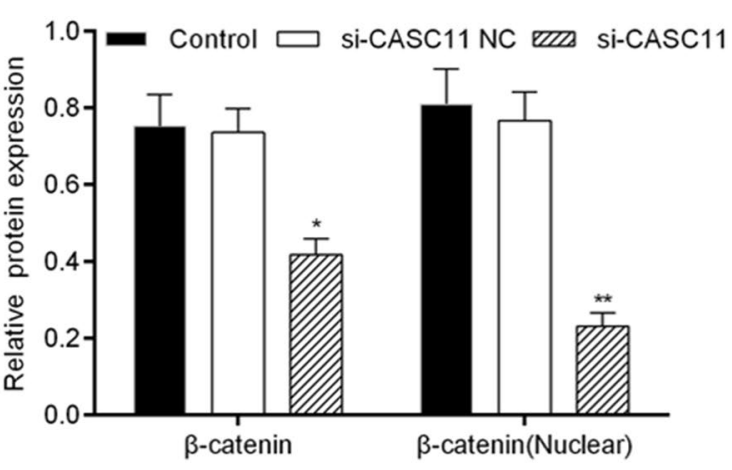

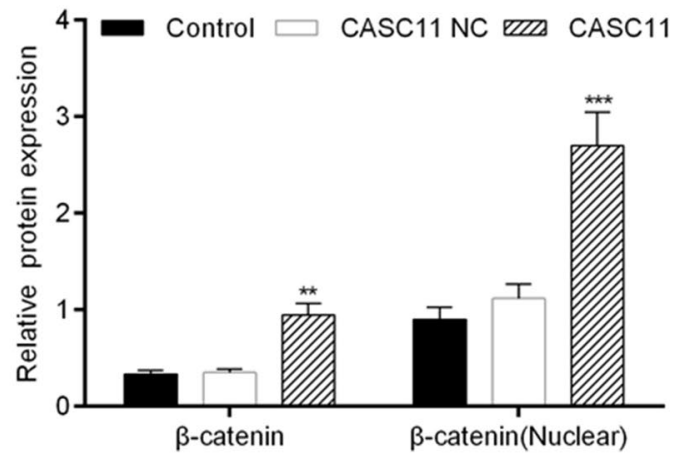

Fig. 5 CASC11 involved in the activating of Wnt/ $\beta$-catenin signaling pathway. a TOP/FOP-Flash luciferase reporter assay was used to analyze the effect of CASC11 on the activity of Wnt/B-catenin signaling pathway in HeLa cells and CaSki cells. b Western blot analysis was used to evaluate the expressions of total and nuclear $\beta$-catenin in si-CASC-transfected HeLa cells and pcDNA 3.1-CASC11-transfected CaSki cells. Data were shown as the mean \pm SD of 3 independent experiments. ${ }^{*} p<0.5 ;{ }^{* *} p<0.01 ;{ }^{* * *} p<0.001$

Because of the high recurrence rate, poor prognosis and current dissatisfactory molecular biomarkers that can predict the prognosis of cervical cancer patients, it is urgent to find new diagnostic and prognostic biomarkers of cervical cancer. In recent years, more and more cervical cancer-related IncRNAs were discovered providing new insights for the treatment of cervical cancer. The lncRNA distal-less homeobox 6 antisense 1 (DLX6$A S 1)$ was reported to be markedly upregulated in cervical cancer tissues and cell lines. Knocking out of DLX6-AS1 inhibited the cell proliferation and induced cell apoptosis by targeting to miR199a [17]. Wang et al. [18] found that BLACAT1 (also known as linc-UBC1) may be served as a novel prognostic biomarker for its positive effects on promoting the proliferation, migration and invasion of cervical cancer cells. However, some lncRNA expressions, for example, IncRNA ZNF667-AS1, have been proved experimentally to be negatively associated with 


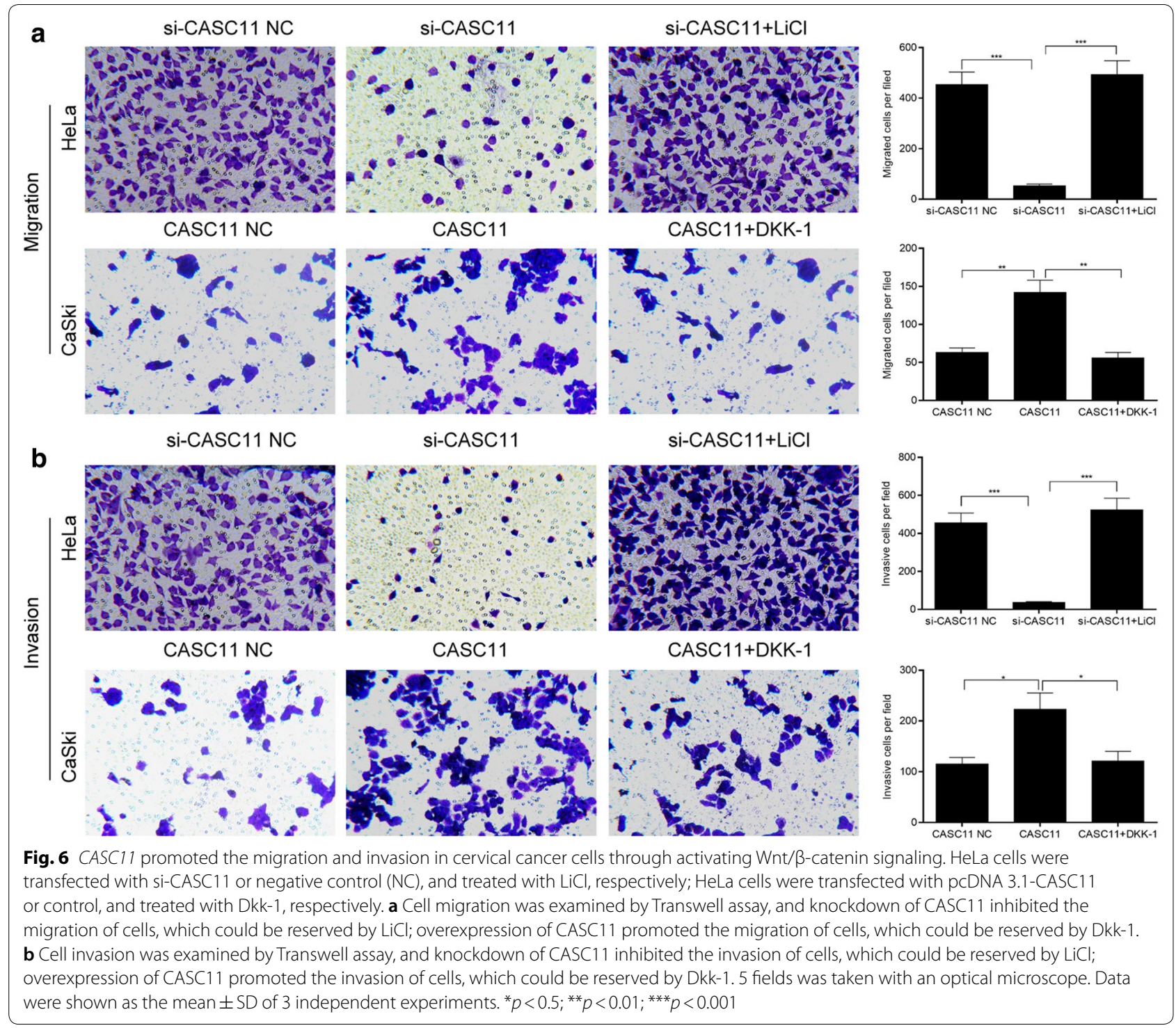

the tumor volumes, FIGO staging and positively related to the patients' survival rate. Overexpression of ZNF667AS1 significantly decreased the proliferation ability and cell cloning ability of HeLa cells [19]. In the present study, we discovered that $C A S C 11$, the expression of which was positively associated with the tumor size and the FIGO staging, was up-regulated in the cervical cancer tissues and cell lines. Kaplan-Meier test showed CASC11 expression was negatively related to the cervical cancer patients' survival rate. Silencing CASC11 by siRNA technology inhibited the proliferation, migration as well as invasion and promoted the cell apoptosis. Conversely, overexpression of CASC11 facilitated the cancer cell growth and suppressed the apoptosis.

Studies have shown that abnormal expressions of signaling molecules including phosphatidylinositol 3-kinase (PI3K), epidermal growth factor receptor (EGF-R), $\beta$-catenin, extracellular signal-regulated kinase (ERK) and $\mathrm{Bcl}-2$ played significant parts in cervical cancer progression [20]. Wnt/ $\beta$-catenin is a canonical pathway that can participate in cell growth, regulation and differentiation. Under normal conditions, $\beta$-catenin is phosphorylated keeping at a low cytoplasmic level at the presence of a GSK3 $\beta$ multiprotein complex. When the signaling pathway is activated, GSK3 $\beta$ kept as an inactive state, which facilitates the cytoplasmic $\beta$-catenin to transfer into the nucleus regulating the expressions of target genes [21]. Many lncRNAs take part in various types cancers through regulating $W n t / \beta$-catenin signaling pathway, like liver cancer, colorectal cancer, breast tumor and so on [5]. Likewise, IncRNAs, such as BLACAT1, LINC00675 


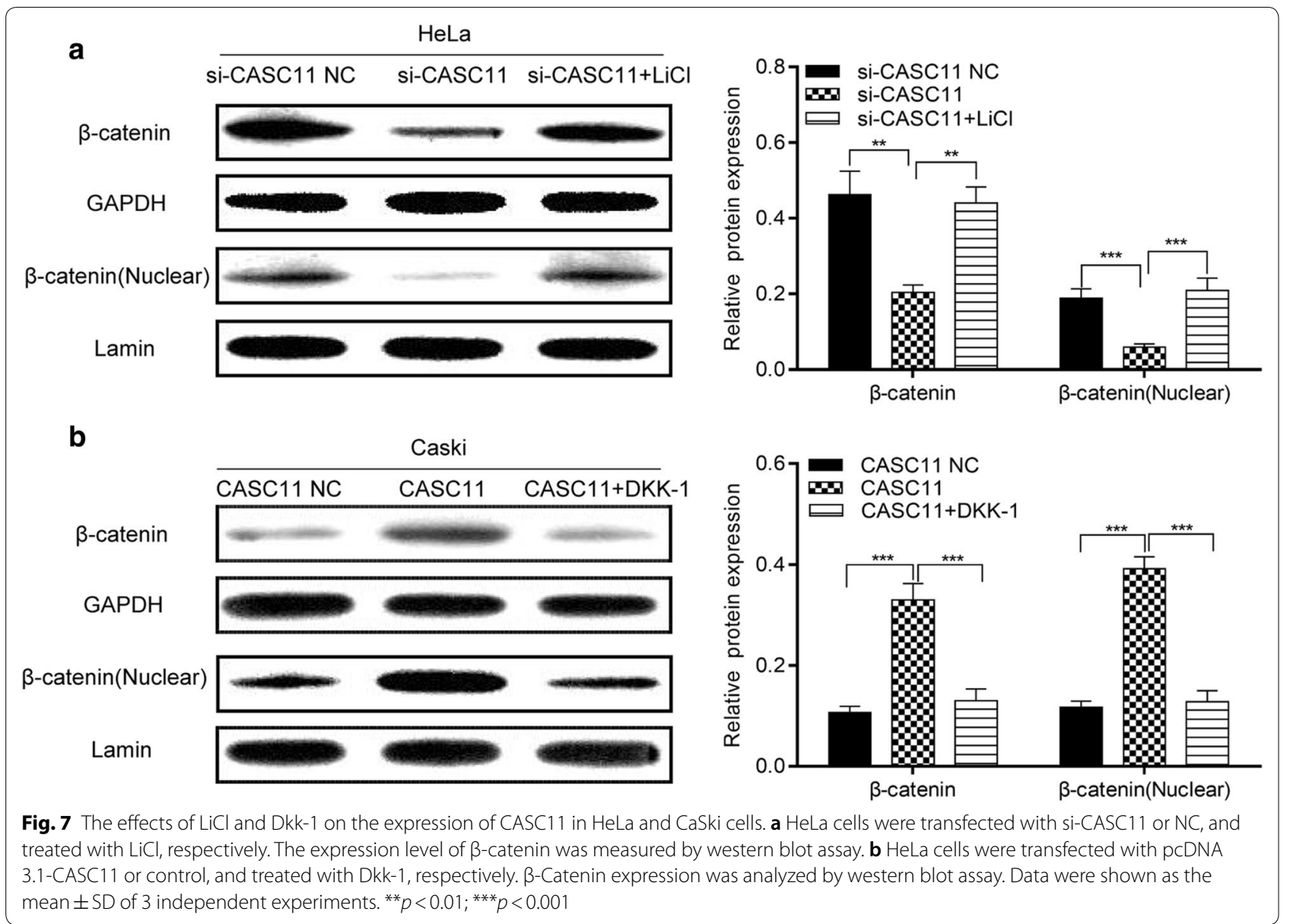

and TUG1, promoted the cervical cancer progression by activating $\mathrm{Wnt} / \beta$-catenin signaling pathway $[18,22$, 23]. In our research, we found si-CASC11 decreased the activity of $\mathrm{Wnt} / \beta$-catenin signaling pathway and the expression of $\beta$-catenin while treatment the siCASC11-transfected cells with $\mathrm{LiCl}$ significantly promoted the migration and invasion of HeLa cells by up-regulating the expression of $\beta$-catenin. Conversely, overexpression of CASC11 increased the activity of Wnt/ $\beta$-catenin signaling pathway and the expression of $\beta$-catenin while treatment the pcDNA3.1-CASC11transfected cells with Dkk-1 significantly depressed the migration and invasion of CaSki cells by reducing the expression of $\beta$-catenin. Effects of CASC11 downregulation and overexpression on migration and invasion and in vivo studies should include the determination of animal metastasis, which should be the focus of future studies. Tumor formation in nude mice showed that inhibition of CASC11 expression decreased the tumor volumes and weight by reducing the expression of $\beta$-catenin. These results indicated that CASC11 promoted the cervical cancer progression by activating $\mathrm{W} n \mathrm{nt} / \beta$-catenin signaling pathway.

\section{Conclusion}

Our study demonstrated that CASC11 promoted the cervical cancer progression by activating Wnt/ $\beta$ catenin signaling pathway for the first time, which provides a new target or a potential diagnostic biomarker of the treatment for cervical cancer. As known to all, lncRNAs function in cancers by interacting with miRNAs, mRNAs and proteins. Therefore, next we will investigate the direct targets of CASC11 in cervical cancers and explore its mechanism comprehensively. In addition, the specific regulatory mechanism of CASC11 on Wnt signaling needs further study in the future. 

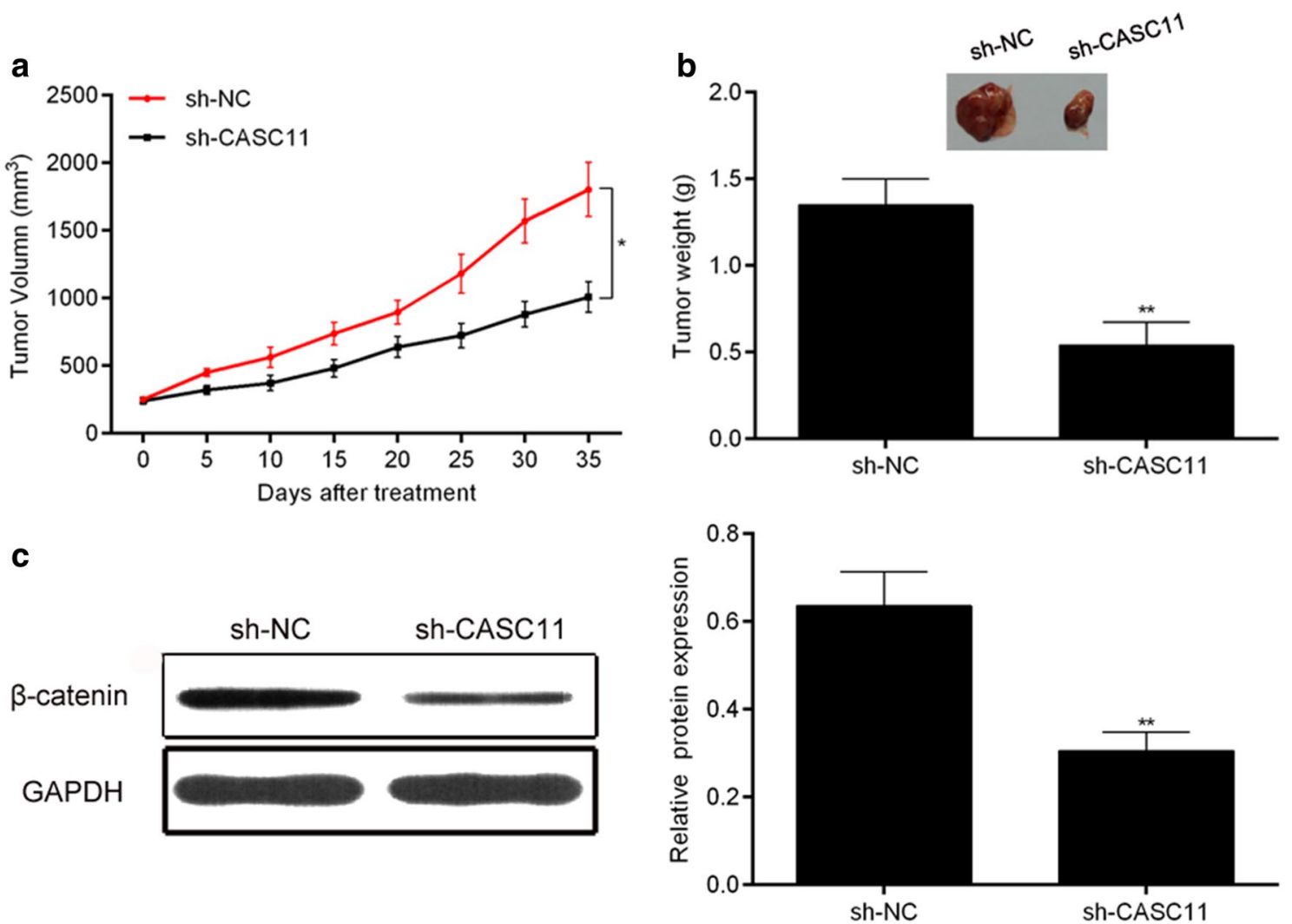

Fig. 8 Knock-down CASC11 inhibited tumor proliferation in vivo. HeLa cells were transfected with sh-CASC11 or sh-NC, respectively. And the cells were injected subcutaneously into nude mice. a Tumor volume was measured, and silencing CASC11 decreased the tumor volume compared to the siRNA NC group. b Tumor weight was measured, and knockdown CASC11 inhibited tumor formation in nude mice ( $n=6$ each group). $\mathbf{c} \beta$-Catenin expression was assessed by western blot assay in the si-NC mice and in the si-CASC11 mice. Data were shown as the mean \pm SD of 3 independent experiments. ${ }^{* *} p<0.01$

\section{Additional file}

Additional file 1: Figure S1. Expression levels of IncRNA CASC11 were determined by RT-qPCR after silencing of si-CASC11-NC, si-CASC11, or overexpression of CASC11-NC, CASC11. Values indicate each point and the average. Values are mean \pm SEM Statistical significance was assessed by the Student's $t$ test. Differences were considered statistically significant. ${ }^{*} p<0.05 ;{ }^{* * *} p<0.001$ ( $\mathrm{n}=3$ per group).

\section{Abbreviations}

CASC11: cancer susceptibility candidate 11; IncRNA: long non-coding RNA; CIN: cervical intraepithelial neoplasia; HPV: human papillomavirus; HOTAIR: HOX transcript antisense RNA; MALAT1: metastasis associated lung adenocarcinoma transcript 1; hnRNP-K: heterogeneous ribonucleoprotein $\mathrm{K}$.

\section{Acknowledgements}

None.

\section{Authors' contributions}

WCH wrote this article; WCH, LFL, XC and YZ participated in the conception and design of the study; WCH and LFL performed the experiments. All authors read and approved the final manuscript.

\section{Funding}

This work was supported by Grants from the Key Talents for Maternal and Child Health of Jiangsu Province (FRC201714) and the National Natural Science Foundation of China (SDFEYGJ1706).

\section{Availability of data and materials}

Source data and material will be made available upon request.

\section{Ethics approval and consent to participate}

All the protocols in this study were approved by the Ethics Committee of the Second Affiliated Hospital of Soochow hospital.

\section{Consent for publication}

Not applicable.

\section{Competing interests}

The authors declare that they have no competing interests.

\section{Author details}

${ }_{1}^{1}$ Department of Gynecology and Obstetrics, The Second Affiliated Hospital of Soochow University, Suzhou 215004, China. ${ }^{2}$ Department of Gynecology and Obstetrics, Xiamen Chang Gung Hospital, Xiamen 361000, China.

Received: 12 January 2019 Accepted: 19 June 2019

Published online: 29 June 2019 


\section{References}

1. Walboomers JM, Jacobs MV, Manos MM, Bosch FX, Kummer JA, Shah $\mathrm{KV}$, et al. Human papillomavirus is a necessary cause of invasive cervical cancer worldwide. J Pathol. 1999;189:12-9.

2. Burd EM. Human papillomavirus and cervical cancer. Clin Microbiol Rev. 2003;16:1-17.

3. Kuglik P, Kasikova K, Smetana J, Vallova V, Lastuvkova A, Moukova L, et al. Molecular cytogenetic analyses of hTERC (3q26) and MYC (8q24) genes amplifications in correlation with oncogenic human papillomavirus infection in Czech patients with cervical intraepithelial neoplasia and cervical carcinomas. Neoplasma. 2015;62:130-9.

4. Sharma S, Mandal P, Sadhukhan T, Chowdhury RR, Mondal NR, Chakravarty B, et al. Bridging links between long noncoding RNA HOTAIR and HPV oncoprotein E7 in cervical cancer pathogenesis. Scientific Rep. 2015;5:11724.

5. Hu X-Y, Hou P-F, Li T-T, Quan H-Y, Li M-L, Lin T, et al. The roles of Wnt/Bcatenin signaling pathway related IncRNAs in cancer. Int J Biol Sci. 2018;14:2003.

6. Chandra Gupta S, Nandan Tripathi Y. Potential of long non-coding RNAs in cancer patients: from biomarkers to therapeutic targets. Int J Cancer. 2017;140:1955-67.

7. Dong J, Su M, Chang W, Zhang K, Wu S, Xu T. Long non-coding RNAs on the stage of cervical cancer. Oncol Rep. 2017;38:1923-31.

8. Xin Y, Li Z, Zheng H, Chan MT, Ka K, Wu W. CCAT 2: a novel oncogenic long non-coding RNA in human cancers. Cell Prolif. 2017;50:e12342.

9. Zhang Z, Zhou C, Chang Y, Zhang Z, Hu Y, Zhang F, et al. Long noncoding RNA CASC 11 interacts with hnRNP-K and activates the WNT/Bcatenin pathway to promote growth and metastasis in colorectal cancer. Cancer Lett. 2016:376:62-73.

10. Han Y, Chen M, Wang A, Fan X. STAT3-induced upregulation of IncRNA CASC11 promotes the cell migration, invasion and epithelial-mesenchymal transition in hepatocellular carcinoma by epigenetically silencing PTEN and activating PI3K/AKT signaling pathway. Biochem Biophys Res Commun. 2019;508:472-9.

11. Zhang L, Kang W, Lu X, Ma S, Dong L, Zou B. LncRNA CASC11 promoted gastric cancer cell proliferation, migration and invasion in vitro by regulating cell cycle pathway. Cell Cycle. 2018;17:1886-900.

12. Ayala-Calvillo E, Mojica-Vázquez LH, García-Carrancá A, González-Maya L. Wnt/ $\beta$-catenin pathway activation and silencing of the APC gene in HPV-positive human cervical cancer-derived cells. Mol Med Rep. 2018;17:200-8.
13. Peng J, Hou F, Feng J, Xu SX, Meng XY. Long non-coding RNA BCYRN1 promotes the proliferation and metastasis of cervical cancer via targeting microRNA-138 in vitro and in vivo. Oncol Lett. 2018:15:5809-18.

14. Lan $K$, Zhao Y, Fan Y, Ma B, Yang S, Liu Q, et al. Sulfiredoxin may promote cervical cancer metastasis via Wnt/B-catenin signaling pathway. Int J Mol Sci. 2017;18:917.

15. Ghoussaini M, Song H, Koessler T, Al Olama AA, Kote-Jarai Z, Driver KE, et al. Multiple loci with different cancer specificities within the $8 \mathrm{q} 24$ gene desert. JNCI J Natl Cancer Inst. 2008;100:962-6.

16. Shen C, Liu Y, Shi S, Zhang R, Zhang T, Xu Q, et al. Long-distance interaction of the integrated HPV fragment with MYC gene and 8q24 22 region upregulating the allele-specific MYC expression in HeLa cells. Int J Cancer. 2017;141:540-8.

17. Wang $X$, Lin Y, Liu J. Long non-coding RNA DLX6-AS1 promotes proliferation by acting as a ceRNA targeting miR-199a in cervical cancer. Mol Med Rep. 2018;19:1248-55.

18. Wang C, Li Y, Tian H, Bao X, Wang Z. Long non-coding RNA BLACAT1 promotes cell proliferation, migration and invasion in cervical cancer through activation of Wnt/ $\beta$-catenin signaling pathway. Eur Rev Med Pharmacol Sci. 2018:22:3002-9.

19. Zhao L, Li R, Han D, Zhang X, Nian G, Wu M, et al. Independent prognostic Factor of low-expressed LncRNA ZNF667-AS1 for cervical cancer and inhibitory function on the proliferation of cervical cancer. Eur Rev Med Pharmacol Sci. 2017;21:5353-60.

20. Yang M, Wang M, Li X, Xie Y, Xia X, Tian J, et al. The role of IncRNAs in signaling pathway implicated in CC. J Cell Biochem. 2018;120:2703-12.

21. Duchartre Y, Kim Y-M, Kahn M. The Wnt signaling pathway in cancer. Crit Rev Oncol Hematol. 2016;99:141-9.

22. Ma S, Deng X, Yang Y, Zhang Q, Zhou T, Liu Z. The IncRNA LINC00675 regulates cell proliferation, migration, and invasion by affecting Wnt/B-catenin signaling in cervical cancer. Biomed Pharmacother. 2018;108:1686-93.

23. Zhu J, Shi H, Liu H, Wang X, Li F. Long non-coding RNA TUG1 promotes cervical cancer progression by regulating the miR-138-5p-SIRT1 axis. Oncotarget. 2017;8:65253.

\section{Publisher's Note}

Springer Nature remains neutral with regard to jurisdictional claims in published maps and institutional affiliations.
Ready to submit your research? Choose BMC and benefit from:

- fast, convenient online submission

- thorough peer review by experienced researchers in your field

- rapid publication on acceptance

- support for research data, including large and complex data types

- gold Open Access which fosters wider collaboration and increased citations

- maximum visibility for your research: over 100M website views per year

At $\mathrm{BMC}$, research is always in progress.

Learn more biomedcentral.com/submissions 